\title{
A weighted eigenvector autofocus method for sparse-aperture ISAR imaging
}

\author{
Jia Duan ${ }^{*}$, Lei Zhang and Meng-dao Xing
}

\begin{abstract}
With the development of multi-functional radar systems, inverse synthetic aperture radar (ISAR) imaging with sparse-aperture (SA) data has drawn considerable attention in the recent years. Motion compensation and imaging are among the most significant challenges that SA-ISAR imaging frequently faces. In this paper, we focus on the autofocus scheme, in which a modified eigenvector-based autofocus method is proposed. In the method, different weights are endued to different range cells according to their signal-to-noise ratios (SNRs). Using the weights, the contribution from the range cells with high SNR is enhanced, yielding accuracy improvement in phase error estimation. What is more is that to improve the estimation precision, an iterative scheme is introduced. Experimental results show that the proposal is not only robust to severe noise but also applicable to ISAR imaging with different SA patterns. Detailed comparisons are given in order to show the superiorities of the proposal in phase adjustment for ISAR data.
\end{abstract}

Keywords: Inverse synthetic aperture radar (ISAR), Autofocus, Sparse aperture (SA), Weighted eigenvector phase adjustment

\section{Introduction}

Inverse synthetic aperture radar (ISAR) has the capability of producing high-resolution images of noncooperative targets in all weather conditions. However, a considerably high-resolution ISAR image is only obtainable when enough pulses are continuously measured. Unfortunately, for a modern radar system, this is difficult: (1) Long coherent processing interval is usually unachievable due to the uncooperative property of ISAR targets. (2) Multi-sourced interferences may contaminate some portions of the received pulse seriously. These portions must be removed in case of false points and blurred images, resulting in discontinuous measurements. (3) With the development of modern multi-mode and multi-functional radar systems, spending long continuous observation time on a single-target measurement is no longer acceptable. Moreover, when multiple targets present simultaneously, the radar systems have to switch among different lines of slight to capture multiple targets. Therefore, for modern radar systems, received

\footnotetext{
* Correspondence: bifiduan119@126.com

National Key Lab of Radar Signal Processing, Xidian University, Xi'an 710071, People's Republic of China
}

pulses for a single target are usually very limited and even discontinuous.

Due to these constraints on data collection, sparseaperture (SA) samples are introduced in modern ISAR systems. Therefore, in order to increase the flexibility and robustness of modern radar systems, the study on sparse-aperture ISAR (SA-ISAR) imaging is urgent. In general, there are two major problems confronting SAISAR imaging.

The first one lies in the way of obtaining full-aperture -resolution ISAR images with SA measurements. To handle this, several methods are proposed, which can be divided into two major categories. The first category is the compressive sensing-based high-resolution SA-ISAR imaging methods [1-6]. These methods are able to achieve an exact or close recovery of signal by solving a minimum $l_{1}$ optimization problem. The other kind of useful technique for full-aperture signal reconstruction is spectral analysis algorithm $[7,8]$. One of the most well-known techniques is the gapped-data amplitude and phase estimation method, which estimates the integrated spectrum by designing narrowband filters to interpolate missing data [8]. 
The second problem confronting SA-ISAR imaging is motion error compensation. Due to the discontinuous sampling process, motion error compensation becomes much more complex in SA-ISAR. Motion error compensation can be achieved by range alignment and phase adjustment. Range alignment is used to remove the range migration of different pulses, while phase adjustment is used to correct their phase errors. It should be emphasized that several existing range alignment methods are already capable to correct range migrations for SA-ISAR data [9-11]. With respect to phase adjustment, there are lots of phase compensation methods available for conventional ISAR. The famous phase gradient autofocus (PGA) method can approximately reach the Cramér-Rao boundary by five or six iterations [12]. The weighted least squares method is a robust algorithm without any requirements on the noise model [13]. However, neither one is suitable for the SA case. To our knowledge, the study on the phase compensation methods for SA-ISAR is quite few. Among the conventional autofocus methods, the eigenvector method is proven to be applicable for SAISAR phase error estimation $[14,15]$. In the eigenvectorbased phase correction method, the maximum likelihood (ML) principle is used, resulting in a solution involving the eigenvector corresponding to the prominent eigenvalue of the sample covariance matrix. Nevertheless, its performance is dependent on the noise level and SA patterns of available samples. To improve the eigenvectorbased phase adjustment, a weighted eigenvector-based phase correction method is addressed in this article. In this method, weights are designed to encourage the contribution of range cells with high signal-to-noise ratio (SNR) and suppress that of range cells with low SNR, yielding a more precise and stable estimation. As an optimal candidate for the SA-ISAR phase adjustment, the weighted eigenvector method performs well in adverse circumstances, such as highly noisy and SA cases. After motion compensation by the proposed autofocus approach, an SA-ISAR imaging method is applied to coherently focus SA-ISAR data for full-aperture-resolution image [3]. The imaging results of simulated and measured data validate the effectiveness of the proposed method.

The remainder of this paper is organized as follows: In the 'Signal model for SA-ISAR' section, the signal model for SA-ISAR is introduced. In the 'Weighted eigenvector method for phase error correction' section, both the theory principle and operation flow of the weighted eigenvector algorithm are illustrated. Moreover, a simple description of the SA-ISAR imaging scheme [3] has been described. In the 'Performance analysis' section, autofocusing and imaging experiments are carried out with acquired ISAR data sets. By comparing with other autofocus algorithms, the superiority of the improved method is shown. Finally, some conclusions are drawn in the last section.

\section{Signal model for SA-ISAR}

Considering that a monostatic ISAR system observes multiple targets simultaneously, the radar has to switch among different targets during the observing time, resulting in SA for each target.

Let $\left\{x_{k}(m)\right\}_{m=1}^{M_{0}}$ denote the $k$ th complete aperture range cell with length $M_{0}$. It can be regarded as a long vector with $P$ sub-apertures,

$$
\boldsymbol{x}_{k} \triangleq\left[\begin{array}{llll}
\boldsymbol{x}_{k}(1) & \boldsymbol{x}_{k}(2) & \cdots & \boldsymbol{x}_{k}\left(M_{0}\right)
\end{array}\right]^{T} \triangleq\left[\begin{array}{llll}
\boldsymbol{x}_{k 1}^{T} & \boldsymbol{x}_{k 2}^{T} & \cdots & \boldsymbol{x}_{k P}^{T}
\end{array}\right]^{T},
$$

where $x_{k 1}^{T}, x_{k 2}^{T}, \cdots, x_{k P}^{T}$ are sub-vectors of $\boldsymbol{x}_{k}$, whose lengths are $L_{1}, L_{2}, \cdots, L_{P}$ respectively, with $L_{1}+L_{2}+\cdots+$ $L_{P}=M_{0}$. Assume that the even sub-apertures of $\boldsymbol{x}_{k}$ are missing due to the radar working mode, and an SA vector $\gamma_{k}$ is formed as follows:

$$
\gamma_{k} \triangleq\left[\begin{array}{llll}
\boldsymbol{x}_{k 1}^{T} & \boldsymbol{x}_{k 3}^{T} & \cdots & \boldsymbol{x}_{k P}^{T}
\end{array}\right]^{T} .
$$

Let $\mu_{k} \triangleq\left[\begin{array}{llll}x_{k 2}^{T} & \boldsymbol{x}_{k 4}^{T} & \cdots & \boldsymbol{x}_{k P-1}^{T}\end{array}\right]^{T}$ denote the missing aperture samples, and $P$ is supposed to be odd for the convenience of the following derivation. Let $\gamma_{k}$ and $\boldsymbol{\mu}_{k}$ have lengths of $M$ and $M_{0}-M$, respectively, where $M$ is the total number of available samples with $M=L_{1}+L_{3}+\cdots L_{P}$.

Figure 1 shows the geometry of the SA-ISAR, in which the full aperture contains $M_{0}$ pulses with an index from 0 to $M_{0}-1$. The odd sub-apertures are available, and the $h$ th sub-aperture consists of $L_{h}$ pulses, whose index is from $N_{h}$ to $N_{h}+L_{h}-1$.

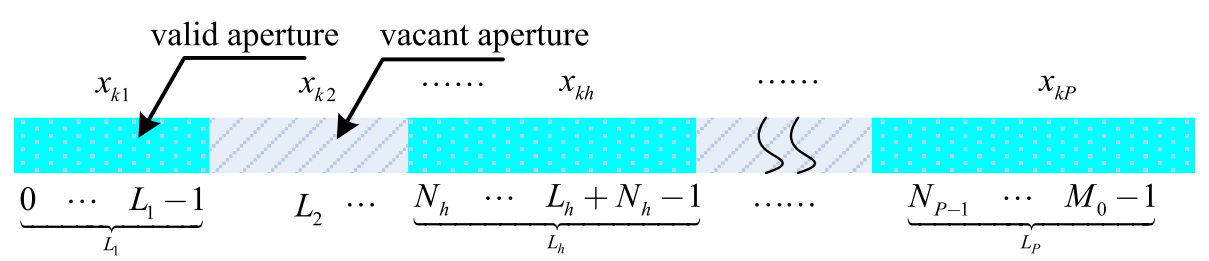

Figure 1 Geometry of sparse-aperture ISAR. 


\section{Weighted eigenvector method for phase error correction}

In conventional ISAR processing, motion compensation usually begins in the range-compressed phase-history domain. Thus, range alignment is implemented to the range-compressed SA data firstly by some novel approaches [9-11], which are proven to be effective even for SA data.

By referring to [14], a signal model for phase error estimation is introduced, in which each dominant range cell contains a single dominant scattering center and other clutters are modeled as uniform-intensity Gaussian white noise. These dominant range cells are usually utilized to estimate phase errors because of their high SNR properties. From [11], a range cell whose normalized amplitude variance is less than $0.12(0.2)$ can be referred to as a dominant range cell.

Hence, the sparsely sampled $l$ th dominant range cell after range compression is given as

$$
\gamma_{l} \triangleq a_{l}\left[\begin{array}{l}
e^{j\left(\varphi_{1}+2 \pi f_{l 1}\right)} \\
\cdots e^{j\left(\varphi_{m}+2 \pi f_{d l} \cdot t_{m}\right)} \ldots e^{j\left(\varphi_{M}+2 \pi f_{d l} \cdot t_{M}\right)}
\end{array}\right]+n_{l},
$$

where $a_{l}$ represents the complex amplitude of the $l$ th dominant range cell; $\boldsymbol{n}_{l}$ denotes the interference from noise, which follows a Gaussian distribution with variance $\delta_{l}^{2}$; Doppler frequency is denoted by $f_{d l}$; and phase error at azimuth position $m$ is $\varphi_{m}$.

Suppose that $L$ dominant range cells are chosen for estimation. For the convenience of deduction, we assume that the Doppler shift of each dominant range cell has been removed temporarily. The Doppler shift removal scheme will be subsequently discussed in the 'Elimination of Doppler shift' section. Then, the rangecompressed dominant echoes can be expressed by

$$
\begin{aligned}
X & =\left[\begin{array}{lllll}
\gamma_{1} & \cdots & \gamma_{l} & \cdots & \gamma_{L}
\end{array}\right]_{M \times L} \\
& =\boldsymbol{v}_{M \times 1} \cdot \boldsymbol{\alpha}_{1 \times L}+\boldsymbol{N}_{M \times L},
\end{aligned}
$$

where the phase error vector is symbolized by $\boldsymbol{v}$ with $\boldsymbol{v}_{M \times 1}=\left[\begin{array}{llll}e^{j\left(\varphi_{1}\right)} & e^{j\left(\varphi_{2}\right)} & \cdots & e^{j\left(\varphi_{M}\right)}\end{array}\right]^{T}$. Note that the phase error is constant across all dominant range cells. The complex amplitude vector is denoted by $\boldsymbol{\alpha}_{1 \times L}$ $=\left[\begin{array}{lll}a_{1} & \cdots & a_{L}\end{array}\right]$, and $N$ stands for the complex Gaussian white noise matrix. Generally, the noise in each dominant range cell is an independently and identically distributed Gaussian random variable. Therefore, we let $\delta_{1}^{2}=\delta_{2}^{2}=\ldots=\delta_{L}^{2}=\delta^{2}$. By this, the covariance matrix of noise can be computed as $\delta^{2} \boldsymbol{I}$ [13], symbolizing as $\operatorname{cov}(\boldsymbol{N})$.

\subsection{Weighted eigenvector method}

\subsubsection{Independent phase error elimination}

Based on the assumption in the last sub-section, the $l$ th dominant range cell for SA-ISAR is expressed as

$$
\begin{aligned}
\gamma_{l} & =a_{l}\left[\begin{array}{llll}
e^{j\left(\varphi_{1}\right)} & e^{j\left(\varphi_{2}\right)} & \ldots & e^{j\left(\varphi_{M}\right)}
\end{array}\right]^{\mathrm{T}}+\boldsymbol{n}_{l} \\
& =a_{l} \boldsymbol{v}_{M \times 1}+\boldsymbol{n}_{l} .
\end{aligned}
$$

Let $\hat{\mathbf{C}}=\frac{1}{L} \boldsymbol{X} \boldsymbol{X}^{H}=\frac{1}{L} \sum_{l=1}^{L} \boldsymbol{\gamma}_{l} \boldsymbol{\gamma}_{l}^{H}$. It has been proven that the ML estimation [9] of $v$ is to choose $v$ maximizing (6) with $\boldsymbol{v}^{H} \boldsymbol{v}=M$,

$$
Q=\boldsymbol{v}^{H} \hat{\boldsymbol{C}} \boldsymbol{v}=\sum_{m=1}^{M} \lambda_{m}\left|z_{m}\right|^{2} .
$$

Define $z$ as $\boldsymbol{z}=\left[\begin{array}{lll}z_{1} & \cdots & z_{M}\end{array}\right]^{T}=\boldsymbol{P}^{H} \boldsymbol{v}$, of which $\boldsymbol{P}$ is the eigenmatrix of $\hat{\boldsymbol{C}}[5]$.

Clearly, (6) is maximized for $z_{\max }$ such that $\left|z_{m}\right|^{2}=M$ and $\left|z_{k}\right|^{2}=0$ for $k \neq m$, where $m$ is the index corresponding to the largest eigenvalue $\lambda_{m}$ of $\hat{\boldsymbol{C}}$. That is, $\boldsymbol{z}_{\max }^{T}=M$. $e^{j \theta}\left[0, \cdots, \frac{1}{m t h}, \cdots, 0\right]$, where $\theta$ is an arbitrary rotation angle. Therefore, the ML estimation of $\boldsymbol{v}$ is $\boldsymbol{v}_{\max }=M \mathrm{e}^{j \theta} \boldsymbol{p}_{m}$, in which $\boldsymbol{p}_{m}$ is the significant eigenvector of $\hat{\boldsymbol{C}}$. After applying $\boldsymbol{p}_{m}$ to correct the phase error, phases are compensated to a constant. Namely, phase adjustment is achieved. In [15], the eigenvector method is validated to be suitable to correcting phase errors for SA-ISAR data.

It should be emphasized that each dominant range cell is fairly treated in the eigenvector-based phase error estimation method. However, for SA-ISAR, there are several dominant range cells with relatively high SNRs. With high SNR property, these dominant range cells are capable of providing more precise information for phase error estimation than others. Based on this, an improved eigenvector autofocus method is established. The improvement involves a weighted optimization function for estimating the phase error. From (6), in order to obtain a more precise estimation, one needs to enhance the contributions of signals and suppress those of noise in the calculation of $\hat{\boldsymbol{C}}$. This can be accomplished by adding significant weights to the dominant range cells with high SNRs, which will be discussed elaborately as follows.

In order to obtain an adjusted $\hat{\boldsymbol{C}}$, we add weights to each dominant range cell. Let $\varepsilon_{l}(l=1,2, \cdots, L)$ represent the weight for the $l$ th cell, then

$$
\boldsymbol{X}^{\prime}=\left[\begin{array}{lll}
\varepsilon_{1} \gamma_{1} & \cdots & \varepsilon_{L} \gamma_{L}
\end{array}\right]_{M \times L}=\boldsymbol{v} \cdot \boldsymbol{a}^{\prime}+\boldsymbol{N}^{\prime},
$$

where the weighted complex amplitude is symbolized by $\boldsymbol{\alpha}^{\prime}$ with $\boldsymbol{\alpha}^{\prime}=\left[\begin{array}{lll}\varepsilon_{1} a_{1} & \cdots & \varepsilon_{L} a_{L}\end{array}\right]$ and weighted noise matrix is denoted by $\boldsymbol{N}^{\prime}=\left[\begin{array}{lll}\varepsilon_{1} \boldsymbol{n}_{1} & \cdots & \varepsilon_{L} \boldsymbol{n}_{L}\end{array}\right]$. The covariance matrix of the weighted noise can be calculated 
with ease, i.e., $\operatorname{cov}\left(\boldsymbol{N}^{\prime}\right)=\frac{1}{L} \sum_{l=1}^{L} \varepsilon_{l}^{2} \delta^{2} \cdot \boldsymbol{I}$. Then, the logarithm of conditional probability density function for the weighted $\boldsymbol{X}^{\prime}$ given $\boldsymbol{v}$ is provided as

$$
\ln p\left(\boldsymbol{X}^{\prime} \mid \boldsymbol{v}\right)=-N \ln \left[\pi^{M}\left|\boldsymbol{C}^{\prime}\right|\right]-\sum_{l=1}^{L} \xi_{l}^{2} \boldsymbol{\gamma}_{l}^{H} \mathbf{C}^{\prime-1} \boldsymbol{\gamma}_{l},
$$

where $C^{n}$ denotes the covariance matrix of the weighted data,

$$
\begin{aligned}
\boldsymbol{C}^{\prime} & =E\left\{\boldsymbol{X}^{\prime} \boldsymbol{X}^{\prime H}\right\} \\
& =\left(\frac{1}{L} \sum_{l=1}^{L} \xi_{l}^{2} a_{l}^{2}\right) \boldsymbol{v} \boldsymbol{v}^{H}+\left(\frac{1}{L} \sum_{l=1}^{L} \xi_{l}^{2} \delta^{2}\right) \boldsymbol{I} .
\end{aligned}
$$

Utilizing $\sigma_{n}^{2}$ to represent $\left(\frac{1}{L} \sum_{l=1}^{L} \xi_{l}^{2} \delta^{2}\right)$ and $\sigma_{a}^{2}$ to represent $\left(\frac{1}{L} \sum_{l=1}^{L} \xi_{l}^{2} a_{l}^{2}\right)$, the inverse matrix of $C^{A}$ is computed as

$$
\left(\boldsymbol{C}^{\prime}\right)^{-1}=-\frac{\sigma_{a}^{2} / \sigma_{n}^{2}}{\sigma_{n}^{2}+M \sigma_{a}^{2}} \boldsymbol{v} \boldsymbol{v}^{H}+\frac{1}{\sigma_{n}^{2}} \boldsymbol{I} .
$$

By substituting (10) into (8), the weighted ML optimal problem has been converted into finding a vector $\boldsymbol{v}$, which maintains the following equation most likely:

$$
Q^{\prime}=\sum_{l=1}^{L} \xi_{l}^{2} \boldsymbol{\gamma}_{l}^{H} \boldsymbol{v} \boldsymbol{v}^{H} \boldsymbol{\gamma}_{l}=\boldsymbol{v}^{H}\left(\sum_{l=1}^{L} \omega_{l} \boldsymbol{\gamma}_{l} \boldsymbol{\gamma}_{l}^{H}\right) \boldsymbol{v}
$$

where $\omega_{l}=\xi_{l}^{2}$. Let $\hat{\boldsymbol{C}}=\frac{1}{L} \sum_{l=1}^{L} \omega_{l} \gamma_{l} \gamma_{l}^{H}$. Similar to the derivation of the eigenvector method, the solution of (11) is related to the eigenvector corresponding to the prominent eigenvalue of $\hat{\boldsymbol{C}}^{\prime}$, after scaling its squared modulus to $M$. Namely, $\hat{\boldsymbol{v}}=M e^{j \theta_{1}} \boldsymbol{p}_{m}{ }^{\prime}$, where $\boldsymbol{p}_{m}{ }^{\prime}$ is the largest eigenvector of $\hat{\boldsymbol{C}}^{\prime}$ and $\theta_{1}$ is an arbitrary rotation angle. Instituting $\hat{\boldsymbol{v}}$ into (11), $Q^{\prime}$ obtains its peak value $\lambda_{\text {max }}^{\prime} M$, where $\lambda_{\text {max }}^{\prime}$ is the largest eigenvalue of $\hat{\boldsymbol{C}}^{\prime}$.

Let $\sum_{l=1}^{L} \omega_{l} \gamma_{l}^{H} \gamma_{l}=\sum_{l=1}^{L} \gamma_{l}^{H} \gamma_{l}$, in that weight does not change the total energy. Here, weight is chosen directly proportional to SNR, which is defined as the ratio of the dominant scatterer energy to the total noise energy in the cell, namely $\omega_{l}=\kappa \frac{a_{l}^{2}}{\delta_{L}^{2}}[16,17]$. A notable point is that the energy of noise includes both that of weak scatterers and clutters. In order to hold the total energy still, $\kappa$ can be normalized as $\Sigma \gamma_{l}^{H} \gamma_{l} / \sum \frac{a_{l}^{2}}{\delta_{L}^{2}} \gamma_{l}^{H} \gamma_{l}$.

For the sake of comparison, the SNR after weighting can be calculated as follows:

$$
\frac{\sum_{l=1}^{L} \omega_{l} a_{l}^{2}}{\sum_{l=1}^{L} \omega_{l} \delta_{l}^{2}}=\frac{\sum_{l=1}^{L} \kappa \frac{a_{l}^{2}}{\delta_{l}^{2}} a_{l}^{2}}{\sum_{l=1}^{L} \kappa \frac{a_{l}^{2}}{\delta_{l}^{2}} \delta_{l}^{2}}=\frac{\sum_{l=1}^{L} \frac{a_{l}^{4}}{\delta_{l}^{2}}}{\sum_{l=1}^{L} a_{l}^{2}}
$$

On the assumption that the noise in each range cell is independently and identically distributed, we have $\delta_{1}^{2}$ $=\delta_{2}^{2}=\ldots=\delta_{L}^{2}=\delta^{2}$. After substituting it into (12), it can be obtained that

$$
\begin{aligned}
\frac{\sum_{l=1}^{L} \omega_{l} a_{l}^{2}}{\sum_{l=1}^{L} \omega_{l} \delta_{l}^{2}}-\frac{\sum_{l=1}^{L} a_{l}^{2}}{\sum_{l=1}^{L} \delta_{l}^{2}}=\frac{\sum_{l=1}^{L} a_{l}^{4}}{\delta^{2} \sum_{l=1}^{L} a_{l}^{2}}-\frac{\sum_{l=1}^{L} a_{l}^{2}}{L \cdot \delta^{2}} \\
=\frac{L \cdot \sum_{l=1}^{L} a_{l}^{4}-\sum_{l=1}^{L} a_{l}^{2} \sum_{l=1}^{L} a_{l}^{2}}{L \cdot \delta^{2} \sum_{l=1}^{L} a_{l}^{2}} .
\end{aligned}
$$

Because of the lemma that $L \sum_{l=1}^{L} b_{l}^{2} \geq \sum_{l=1}^{L} b_{l} \sum_{l=1}^{L} b_{l}$, we have $L \cdot \sum_{l=1}^{L} a_{l}^{4}-\sum_{l=1}^{L} a_{l}^{2} \sum_{l=1}^{L} a_{l}^{2} \geq 0$. The detailed derivation of the lemma is listed as follows:

$$
\begin{aligned}
\sum_{l=1}^{L} b_{l} \sum_{l=1}^{L} b_{l}^{l} & =\sum_{i=1}^{L} \sum_{j=1}^{L} b_{i} b_{j} \leq \sum_{i=1}^{L} \sum_{j=1}^{L} \frac{b_{i}^{2}+b_{j}^{2}}{2} \\
& =\sum_{i=1}^{L}\left(\frac{L}{2} b_{i}^{2}+\left(\frac{1}{2}\right) \sum_{j=1}^{L} b_{j}^{2}\right) \\
& =\frac{L}{2} \sum_{i=1}^{L} b_{i}^{2}+\frac{L}{2} \sum_{j=1}^{L} b_{j}^{2}=L \sum_{l=1}^{L} b_{l}^{2} .
\end{aligned}
$$

After substituting $L \cdot \sum_{l=1}^{L} a_{l}^{4}-\sum_{l=1}^{L} a_{l}^{2} \sum_{l=1}^{L} a_{l}^{2} \geq 0$ into (13), we have $\frac{\sum_{l=1}^{L} \omega_{l} a_{l}^{2}}{\sum_{l=1}^{L} \omega_{l} \delta_{l}^{2}} \geq \frac{\sum_{l=1}^{L} a_{l}^{2}}{\sum_{l=1}^{L} \delta_{l}^{2}}$. Therefore, the SNR of $\hat{C}^{\prime}$ has been improved by the weighting processing compared with that of $\hat{\boldsymbol{C}}$. In this way, the maximum eigenvalue $\lambda_{\text {max }}^{\prime}$ of $\hat{\boldsymbol{C}}^{\prime}$ becomes more significant than that of $\hat{\boldsymbol{C}}$, yielding an optimized ML estimation. Furthermore, enhanced signal components and suppressed noise components are achieved using the SNR weights. Compared 
with (6), a more precise phase adjustment is resulted in than before.

\subsubsection{Elimination of Doppler shift}

On the assumption that the Doppler shift of each dominant range cell is removed, the weighted eigenvectorbased autofocus method is effective. However, the Doppler shift is relevant to the cross-range position of each dominant scattering center, which differs with both azimuth and range positions. So as to ensure the feasibility of our method, Doppler shift should be removed before the coherence matrix calculation.

In traditional ISAR phase error compensation methods, center shifting is utilized to reduce the influence of Doppler shifts, which works in two steps. Firstly, by Fourier transforming (FT), echoes are transformed into the range-Doppler (RD) image domain. Then, Doppler shift is removed by circularly removing each dominant scatterer to the center of the image. However, for SAISAR, especially when it comes to the unevenly undersampling cases, the method needs some modification. Because of the discontinuous phase history, it is impossible for the SA-ISAR signal to be coherently accumulated by FT. To handle this, zero padding is utilized [13]. In this way, FT can be useful, namely circularly shifting can be implemented. In this article, instead of circularly shifting, we multiply a corresponding linear-phase function with the zero-padded signal in the time domain. The linearphase function is constructed by estimating Doppler shifts from the positions of strongest response in the RD domain. Note that although the sidelobes are highly raised, the mainlobe is still higher so that the positions can be determined. Finally, vacant apertures are removed from the product.

Nonetheless, due to the presence of phase error, the Doppler shift removal is not optimal as expected. Therefore, the phase error correction and Doppler shift estimation are done in a mutually iterative manner, in which one estimated variable is used to update the estimation of the other. Through this scheme, the Doppler shift and phase error are estimated and reduced gradually. Finally, with the increase of iteration number, precise estimations are resulted in. For clarity, the flowchart of the weighted eigenvector autofocus method is shown in Figure 2.

Firstly, in order to determine which range cells are chosen as the samples for coherence matrix computation, we compute the normalized amplitude variance of each range cell.

Secondly, the estimation of phase errors is done in two steps:

- Step 1: Weight is calculated for each dominant range cell according to SNR. Then, the weighted covariance matrix is obtained, and the eigenvector corresponding to the largest eigenvalue is used to correct the phase errors roughly. Subsequently, Doppler shift is estimated and compensated.

- Step 2: Re-determine phase errors by the weighted eigenvector autofocus method. After compensating the estimated phase error to the dominant range cells, Doppler shift is re-estimated and compensated.

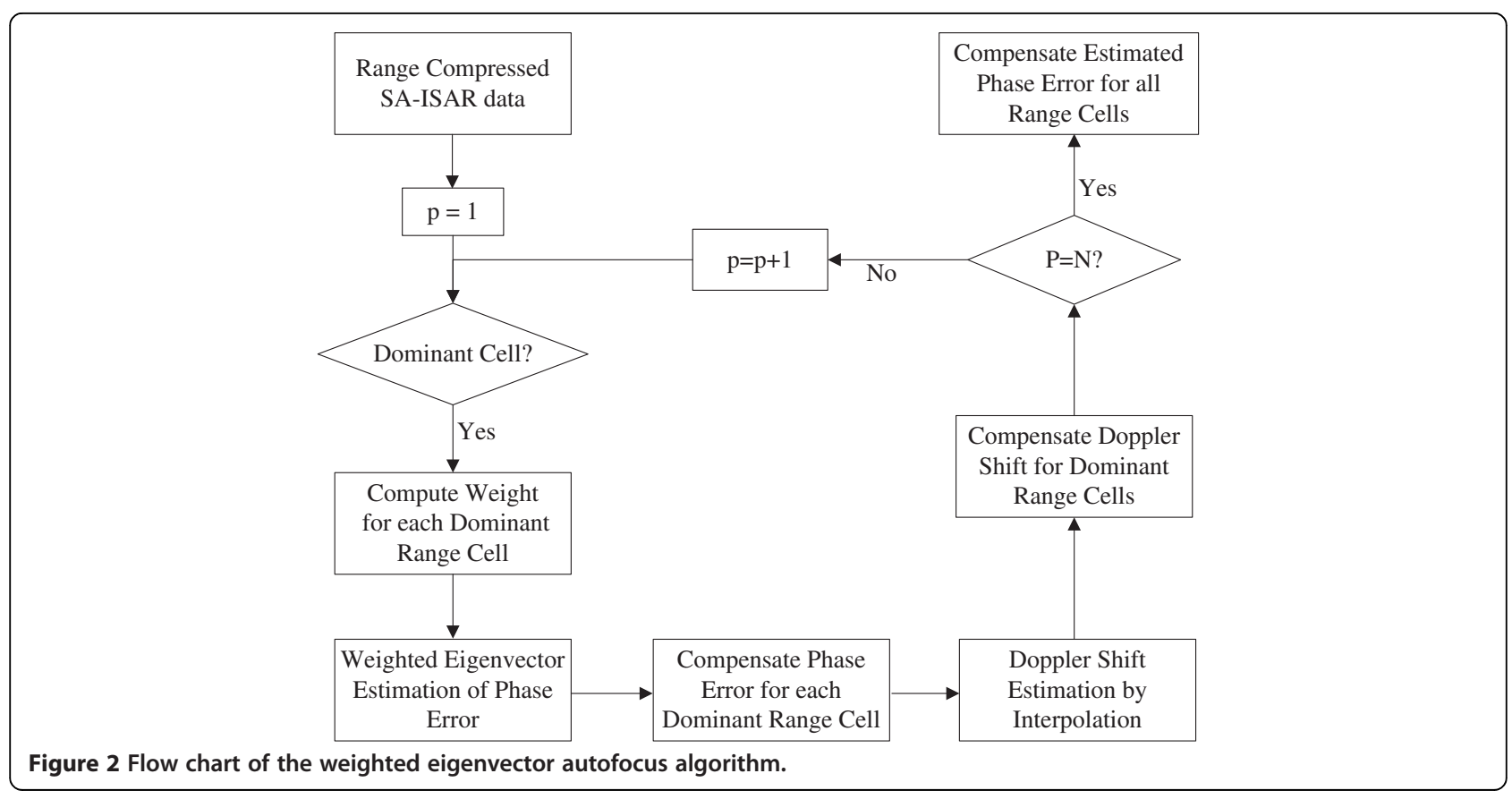


Continue the above sub-steps until the ceasing condition is satisfied. The halt condition may be the count of iteration number. Experimental results show that two or three iterations are enough to ensure the accuracy.

At last, we compensate the whole range cells with the estimated phase error.

\subsection{SA-ISAR imaging}

For traditional ISAR after autofocusing, RD imaging is a simple but effective method to obtain a well-focused image of targets. However, for SA-ISAR data with the sparsely sampled Doppler history, RD images of targets usually smear seriously with high gratinglobes and sidelobes. To reduce the discontinuous sampling effects on SA imagery, many novel approaches have been proposed. In [3], a sparsity-driven algorithm to generate high-resolution SA-ISAR images is given, in which a SAISAR imaging problem is converted into a sparsityconstrained optimization problem based on the maximum a posteriori estimation. The optimization is given as follows:

$$
\begin{aligned}
\hat{A}(\boldsymbol{X}) & =\arg \max _{\mathrm{A} \in \mathrm{C}_{N \times M}}\left\{-\frac{1}{2 \sigma^{2}}\|\boldsymbol{X}-\boldsymbol{F} \boldsymbol{A}\|_{2}^{2}-\gamma\|\boldsymbol{A}\|_{1}\right\} \\
& =\arg \min _{\mathrm{A} \in \mathrm{C}_{N \times M}}\left\{\|\boldsymbol{X}-\mathrm{F} \boldsymbol{A}\|_{2}^{2}+\mu\|\boldsymbol{A}\|_{1}\right\},
\end{aligned}
$$

where $\mu=2 \sigma^{2} \gamma$ is the sparsity coefficient and $\gamma$ is the Laplace distribution parameter. They are directly related to the unknown statistics of noise and target signal. $X$ stands for the SA-ISAR echo matrix, as defined before. $F$ is a partial Fourier matrix, which can be easily constructed corresponding to the pattern of SA. $\boldsymbol{A}=\left[a_{n m}\right]$ is an $N \times M$ matrix and denotes the two-dimensional (2D) ISAR image, whose pixel values are corresponding to scattering center amplitudes.

Based on the assumption that the additive noise is subject to a zero-mean Gaussian distribution with unknown variance $\sigma^{2}$ and the signal components corresponding to the dominant scattering centers follow a Laplace distribution with coefficient $\gamma$ independently, it utilizes the constant-false-alarm-ratio detector to discriminate signal from noise in the sub-aperture images approximately. Using the pure noise and target components, both $\sigma^{2}$ and $\gamma$ can be obtained via ML. In this SA-ISAR imaging algorithm, a modified quasi-Newton algorithm is applied in an iterative manner for image formation [4], and in order to improve the efficiency of the solver, fast Fourier transform and conjugate gradient algorithm are applied in its implementation [3]. Real data experiments manifest the effectiveness of the method. Therefore, we use this SA-ISAR imaging algorithm jointed with phase adjustment proposed to achieve a high-quality SA-ISAR image.

\section{Performance analysis}

In this section, real ground-based measurements are conducted to analyze the performance of the weighted eigenvector phase correction method. By considering different SA patterns, such as complete aperture pattern, unevenly SA pattern, and block SA pattern, the universality of our method is investigated. The following experiments are vital to validate the effectiveness of our method.

\subsection{Dataset and evaluation criterion}

To make our experiments convincing, real measured ISAR data are used to perform different experiments. A dataset of Yak-42 airplane is used, which is recorded using a C-band $(5.52 \mathrm{GHz})$ ISAR experimental system. This system transmits a $100-\mathrm{MHz}$ linear modulated chirp signal with $25.6-\mu$ s pulse width. The range resolution is $0.375 \mathrm{~m}$. The echo is de-chirped and I/Q sampled for range compression. After range compression, conventional range alignment and phase adjustment are applied to the complete aperture data. Then, RD imaging method is implemented, producing a well-focused image, as illustrated in Figure 3. We use it as a standard image for evaluating the following experimental results.

After applying 2D inverse Fourier transforming to the well-focused image, random Gaussian phase error and white noise are added to generate defocused data sets with different SNRs. The added phases randomly vary within $[-\pi, \pi]$. Moreover, SA-ISAR data are created by extracting some pulses from the data sets in both random and block manners. Thus, the performance of phase correction methods under different conditions is analyzed.

In order to assess the performance quantitatively, criteria should be built. Since the entropy of an image is usually used as an index that quantitatively represents the information of the luminosity distribution of the

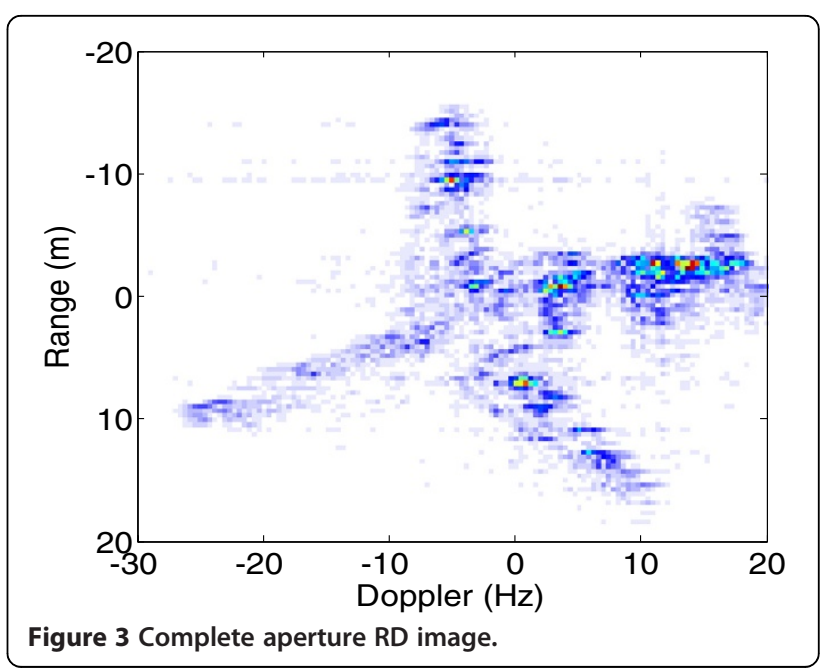


image, entropy criterion is used to analyze the performance for the complete aperture case. However, owing to the use of parametric superresolution approaches, the image entropy criterion is not suitable to SA-ISAR images [4]. Therefore, a criterion built in [13] is brought in to appraise the weighted eigenvector phase adjustment method for SA cases quantitatively:

$$
p_{e}=\frac{1}{M} \sum_{m=1}^{M}|v(m)-\hat{v}(m)|^{2}
$$

where $p_{\mathrm{e}}$ is defined as the square error between estimated phase and added phase, and $v$ and $\hat{v}$ are the applied and estimated phase errors, respectively.

\subsection{Experimental results and analysis \\ 4.2.1. Performance under low SNR with complete aperture data}

To testify the effectiveness of our method under low SNR, complex-valued Gaussian noise and phase error are added to generate degraded data set with $0-\mathrm{dB}$ SNR. After range compression, three autofocus methods (traditional eigenvector method, PGA algorithm, and weighted eigenvector method) are performed to estimate the phase errors. Since we use complete aperture here, traditional $\mathrm{RD}$ imaging algorithm is adopted. The RD images with different phase adjustment methods (PGA, eigenvector, and weighted eigenvector methods) are shown in Figure 4a,b,c, respectively. Note that although the original eigenvector method can get highly focused results in certain range cells, blurring cases emerge in
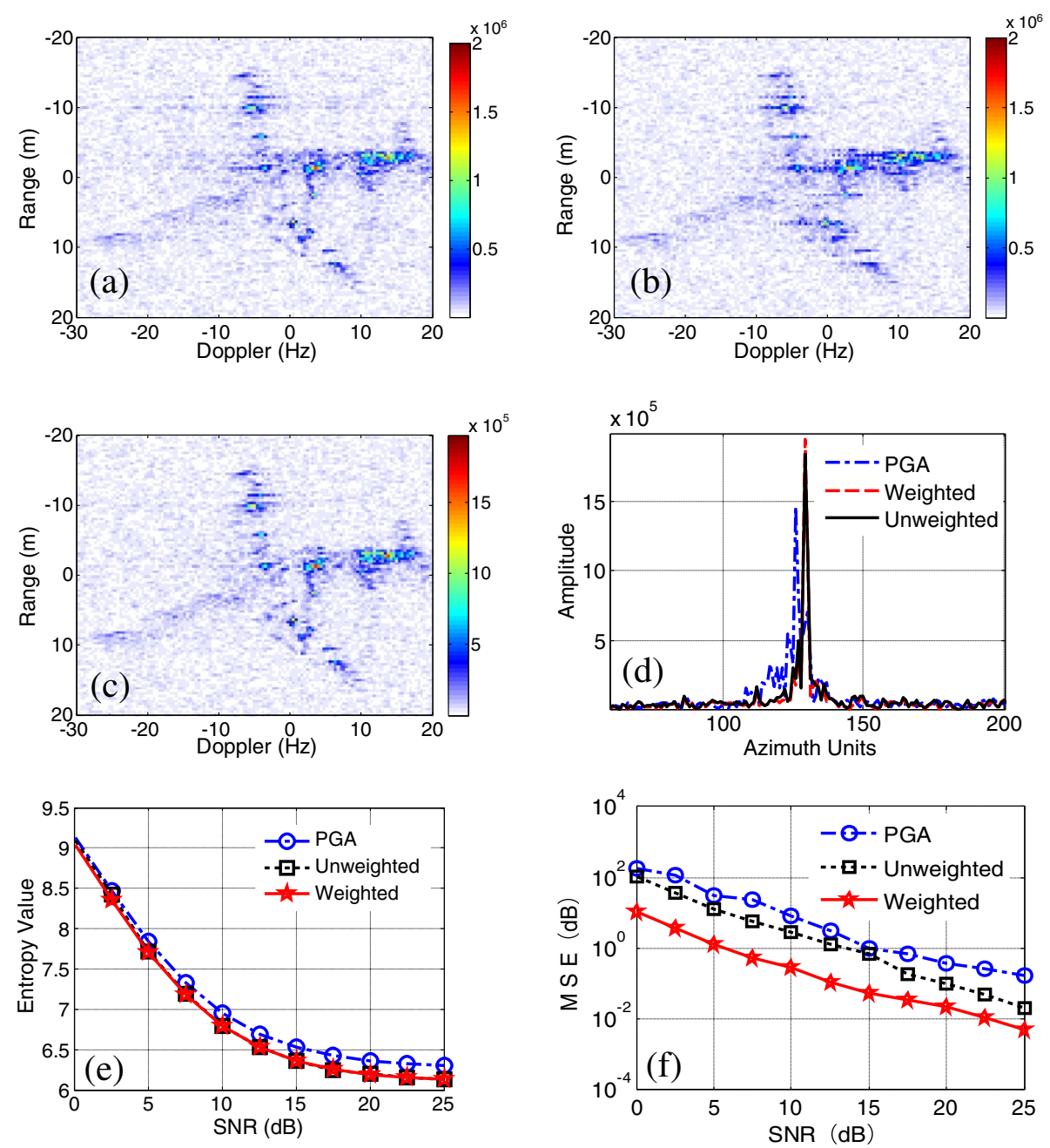

Figure 4 Imaging results for complete aperture data under low SNRs. (a) Eigenvector method. (b) PGA algorithm. (c) Weighted eigenvector method. (d) Envelope of the 146th range unit. (e) Average entropy value under different SNRs. (f) Entropy MSE under different SNRs. 


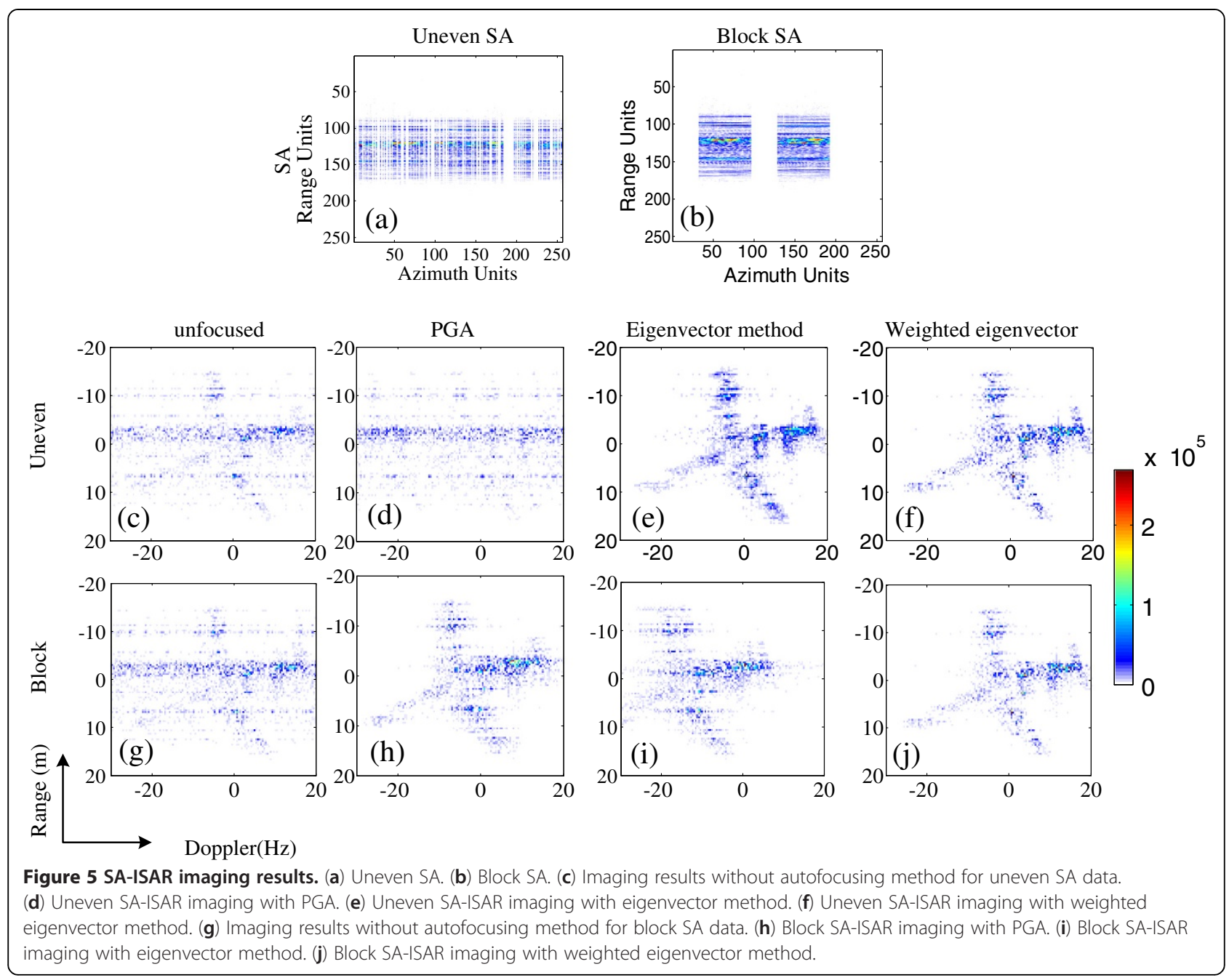

other range cells. This is improved by the weighted eigenvector method, which is better than the result of PGA as well.

For comparison, the 146th range cell with one dominant scatterer is chosen. The envelope of the 146th range cell is plotted in Figure $4 \mathrm{~d}$. It is obvious that the mainlobe of the proposed algorithm is the highest and the sidelobe is the lowest of the three methods. Therefore, the weighted method outperforms other methods under 0-dB SNR.

Furthermore, the performance of the three phase adjustment methods under different SNRs is analyzed and compared by Monte Carlo simulation. At each generated SNR, a hundred independent experiments have been conducted. Entropy of the focused image is computed. Its definition is given in [18], which is

$$
\begin{aligned}
& u=\sum_{m} \sum_{n} \frac{|A(m, n)|^{2}}{S} \ln \frac{S}{|A(m, n)|^{2}} \\
& S=\sum_{m} \sum_{n}|A(m, n)|^{2}
\end{aligned}
$$

where $u$ means the entropy value of an ISAR image, $A$ $(m, n)$ is the amplitude of pixel $(m, n)$, and $S$ is the total energy of the ISAR image.

The mean value of entropy with SNR is presented in Figure 4e. From the image, the proposed method is superior to others, especially under low SNRs. With the increased SNR, the entropy values decrease sharply and results of the three methods become similar. As to the comparison of the steadiness, Figure $4 \mathrm{f}$ shows the mean square error (MSE) of entropy varying with SNR, with which the results indicate that the proposed method is robust and stable under low SNR conditions. The definition of MSE has been given as

Table 1 Mean square error between true phase error and estimated error

\begin{tabular}{lccc}
\hline & PGA & Eigenvector & Weighted eigenvector \\
\hline Unevenly SA & 0.2431 & 0.1054 & 0.0437 \\
Block SA & 0.1928 & 0.0967 & 0.0587 \\
\hline
\end{tabular}




$$
M S E=\frac{1}{K} \sum_{k=1}^{K}|u(k)-\bar{u}|^{2},
$$

where $K$ is meant by the total number of Monte Carlo experiments under each generated SNR, $u(k)$ represents the entropy value of the SA-ISAR imagery in the $k$ th experiment, and $\bar{u}$ is the average entropy value of total independent experiments under certain SNR.

\subsubsection{Performance under SA cases}

In this sub-section, random Gaussian phase error is applied to defocus the dataset, and 128 pulses are extracted from the degraded dataset as SA samples. The SAs are illustrated in Figure 5a,b, respectively. The under-sampling processes are done in two ways, unevenly under-sampling and block under-sampling. Uneven SA is a general SA case, in which the missing data can occur at an arbitrary sampling index, and block SA is a familiar SA pattern in multiple-target observation with a single radar system. In this SA pattern, discontinuous blocks constitute the SA data, with each block having no missing samples.

Firstly, the sparsity-driven SA-ISAR imaging method is implemented to the range-compressed data without any autofocus methods. The results are presented in Figure 5c, $\mathrm{g}$, respectively. The images are defocused in both SA cases. To autofocus the SA data, three methods (PGA, eigenvector method, and weighted eigenvector method) are conducted. The imaging results manifest that the weighted eigenvector method achieves well-focused results under both SA patterns, as shown in Figure 5. However, the eigenvector method is only adaptable for the unevenly SA case, while the PGA is not suitable for both SA cases. Therefore, we conclude our method is suitable for autofocusing data with different SA patterns, which is an extraordinary superiority to autofocusing methods available.

For quantitative comparison, the square error criterion given in [13] is adopted. Square error is computed according to (16) with the solutions listed in Table 1. Apparently, the weighted eigenvector has the smallest square errors in both SA patterns, which accords with the imaging results.

\section{Conclusions}

Based on the traditional eigenvector method, a weighted method is proposed. After comparing with traditional autofocus methods, such as PGA in conventional ISAR, it has been proven that the proposed method can achieve a well-focused SA-ISAR image, especially under low SNR conditions. With respect to the case that center shifting is not suitable for the SA-ISAR, zeropadding and iteration are implemented to eliminate discrete Doppler shift. Finally, the experimental results of real acquired data have testified that the proposed method is capable to correcting phase error for SA-ISAR data with any pattern, whether block sparse-sampled or unevenly sparse-sampled data sets.

\section{Competing interests}

The authors declare that they have no competing interests.

\section{Acknowledgments}

The authors thank the anonymous reviewers for their valuable comments to improve the paper quality. This study was supported by the National Natural Science Foundation of China under grant nos. 61222108, JJ0200122201, and 61179010 and by the Fundamental Research Funds for the Central Universities under grants K5051302001 and K5051302038.

Received: 6 May 2012 Accepted: 1 April 2013

Published: 29 April 2013

\section{References}

1. L Zhang, M Xing, C Qiu, J Li, Z Bao, Achieving higher resolution ISAR imaging with limited pulses via compressed sampling. IEEE GRSL. 6(3), 567-571 (2009)

2. H Joachim, G Ender, On compressive sensing applied to radar. IEEE Trans. SP. 90(5), 1402-1414 (2010)

3. L Zhang, Z Qiao, M Xing, J Sheng, R Guo, Z Bao, High resolution ISAR imaging by exploiting sparse apertures. IEEE Trans. Ant. Pro. 60(2), 997-1008 (2012)

4. L Zhang, MD Xing, CW Qiu, J Li, J Sheng, Y Li, Z Bao, Resolution enhancement for inversed synthetic aperture radar imaging under low SNR via improved compressive sensing. IEEE Trans. GRS. 48(10), 3824-3838 (2010)

5. SD Babacan, R Molina, AK Katsaggelos, Bayesian compressive sensing using Laplace priors. IEEE Trans. IP. 19(1), 53-63 (2010)

6. JA Tropp, AC Gilbert, Signal recovery from random measurements via orthogonal matching pursuit. IEEE Trans. IT. 53(12), 4655-4666 (2007)

7. J Capon, High resolution frequency-wavenumber spectrum analysis. Proc. IEEE. 57, 1408-1418 (1969)

8. J Li, P Stocia, An adaptive filtering approach to spectral estimation and SAR imaging. IEEE Trans. Signal Process 44(6), 1469-1484 (1996)

9. J Wang, D Kasilingam, Global range alignment for ISAR. IEEE Trans. Aerosp. Syst. 39(1), 351-357 (2003)

10. T Sauer, A Schroth, Robust range alignment algorithm via Hough transform in an ISAR imaging system. IEEE Trans. Aerosp. Electron. Syst. 31(3), 1173-1177 (1995)

11. D Zhu, L Wang, Y Yu, Q Tao, Z Zhu, Robust ISAR range alignment via minimizing the entropy of the average range profile. IEEE. Geosci. Remote Sens. Lett. 6(2), 204-208 (2009)

12. DE Wahl, PH Eichel, DC Ghiglia, CV Jakowatz, Phase gradient autofocus - a robust tool for high resolution SAR phase correction. IEEE Trans. Aerosp. Electron. Syst. 30(3), 827-834 (1994)

13. W Ye, TS Yeo, Z Bao, Weighted least-square estimation of phase errors for SAR ISAR autofocus. IEEE Trans. Geosci. Remote Sens. 37(5), 2488-2494 (1999)

14. CV Jakowatz Jr., DE Wahl, Eigenvector method for maximum-likelihood estimation of phase errors in synthetic-aperture-radar imagery. J. Optical Soc. Am. 10(12), 2539-2546 (1993)

15. D Zhu, X Yu, Z Zhu, Algorithms for compressed ISAR autofocusing, in Proceedings of the 2011 IEEE CIE International Conference on Radar. Chengdu 24-27, 533-536 (Oct 2011)

16. MA Win, JH Winters, Analysis of hybrid selection/maximal-ratio combining in Rayleigh fading. IEEE Trans. Communications 47(12), 1773-1776 (1999)

17. N Kong, LB Milstein, Average SNR of a generalized diversity selection combining scheme. IEEE. Communication Letters 3(3), 57-60 (1999)

18. J Wang, X Liu, Z Zhou, Minimum-entropy phase adjustment for ISAR. IEE. Proc. Radar Sonar Navig. 15(4), 203-209 (2004)

doi:10.1186/1687-6180-2013-92

Cite this article as: Duan et al:: A weighted eigenvector autofocus method for sparse-aperture ISAR imaging. EURASIP Journal on Advances in Signal Processing 2013 2013:92. 